\title{
Escherichia Coli Subscapular Abscess as a Rare Complication of Manual Therapy in Frozen Shoulder: A Case Report
}

\section{Dong Hun Ham}

Department of Orthopeadic Surgery, St. Carollo Hospital

\section{Sungil Wang ( $\sim$ wsi1205@naver.com )}

Chonbuk National University Hospital: Jeonbuk National University Hospital https://orcid.org/00000002-3890-6516

\section{Case report}

Keywords: Abscess, Subscapularis, Escherichia coli, Manual therapy

Posted Date: January 5th, 2021

DOI: https://doi.org/10.21203/rs.3.rs-138796/v1

License: (c) (i) This work is licensed under a Creative Commons Attribution 4.0 International License.

Read Full License 


\section{Abstract}

\section{Background:}

Subscapular abscess is a rare condition usually secondary to immunocompromised state and recent infection. Staphylococcus aureus (S. aureus) is the most common etiologic agent. To the best of our knowledge, we present the first case of a patient with a frozen shoulder performing manual therapy, which resulted in an Escherichia coli subscapular abscess.

Case presentation: A 72-year-old male was referred from the respiratory department with pain and a limited range of motion in the left shoulder. He complained that the pain was exacerbated with popping sound during manual therapy for frozen shoulder two weeks ago. Magnetic resonance imaging revealed a large intramuscular complicated fluid collection at the level of the subscapularis muscle $10.0 \times 5.2 \times 11$ $\mathrm{cm}$ in size, and a bloody but turbid pus-like discharge was observed upon aspiration. He underwent urgent surgical drainage. The cultures from the abscess fluid revealed extended-spectrum betalactamases (-) E. coli. After 4 weeks of Ceftazidime intravenous treatment, symptoms of the patient had improved.

Conclusions: This case highlights the risks of aggressive manual therapy in severe frozen shoulder. It also presents a clinical scenario in which practitioners of manual therapy in frozen shoulder should be aware of the risk of subscapular abscess in patients undergoing recent infection therapy

\section{Background}

Subscapular abscess is an uncommon condition. Predisposing conditions include an immunocompromised state, recent infection, diabetes, end-stage renal disease, intravenous (IV) drug use, and trauma to the shoulder area causing hematoma formation [1-4]. Staphylococcus aureus (S. aureus) is the most common etiologic agent [1-8], and other less-common pathogens include Haemophilus Influenzae (H. influenza) [9]. However, there are no reported cases of Escherichia coli (E. coli) causing a subscapular abscess.

Meanwhile, manual therapy and exercise, usually delivered together as components of a physical therapy intervention, are commonly used interventions for adhesive capsulitis. Manual therapy includes any clinician-applied movement of the joints and other structures, for example, mobilization or manipulation. Although manual therapy and stretching exercises have been demonstrated to reduce pain and improve function in a frozen shoulder [10], few reports of complications related to this for frozen shoulder.

Here, we report the first case of an E. coli subscapular abscess that occurred after manual therapy for the treatment of frozen shoulder in a 72-year-old man.

\section{Case Presentation}


A 72-year-old male was referred from the respiratory department with pain and a limited range of motion in the left shoulder. He complained that the pain was exacerbated with popping sound during manual therapy for frozen shoulder two weeks ago. A review of his medical history indicated that he was treated with intravenous (IV) antibiotics for 6 weeks in the respiratory intensive care unit of our hospital for atypical pneumonia 2 months ago.

Examination done at the time of admission to our clinic revealed swelling in the anterior aspect of the shoulder and axillary regions. The patient presented with a mild fever of $37.8^{\circ} \mathrm{C}$. In laboratory tests, his white-cell count was $4.69 \times 10^{3} / \mu \mathrm{l}$ (normal $4.8-10.8 \times 10^{3} / \mu \mathrm{l}$ ), neutrophil cell count was $51.8 \%$ (normal $50-75 \%$ ), erythrocyte sedimentation rate was $28 \mathrm{~mm} / \mathrm{h}$ (normal 0-9 mm/h), and C-reactive protein was $81.54 \mathrm{mg} / \mathrm{L}$ (normal $0-5 \mathrm{mg} / \mathrm{L}$ ). The plain radiographs of the left shoulder were normal. Chest computed tomography showed a newly observed lesion with thickened walls in the left subscapularis muscle, including fluid in an area of $10 \times 5 \mathrm{~cm}$ (Fig. 1A and 1B). Subsequent sequential magnetic resonance imaging (MRI) revealed a large intramuscular complicated fluid collection at the level of the subscapularis muscle $10.0 \times 5.2 \times 11 \mathrm{~cm}$ in size (Fig. 2A and 2B), and a bloody but turbid pus-like discharge was observed upon aspiration (Fig. 3).

Accordingly, we did surgical drainage via a standard deltopectoral approach. During mobilization of the conjoined tendon, a large amount of blood-stained pus exuded from the subscapularis muscle. The cultures from the abscess fluid revealed extended-spectrum beta-lactamases (-) E. coli, susceptible to ceftazidime (minimum inhibitory concentration $\leqq 1 \mu \mathrm{g} / \mathrm{mL}$, as well as to nine other tested antibiotics, and resistant to ampicillin and sulbactam $(>32 \mu \mathrm{g} / \mathrm{mL})$. Blood cultures obtained the time of admission also revealed extended-spectrum beta-lactamases (-) E. coli. However, urinalysis and urine culture results were negative. Ceftazidime IV treatment was administered based on consultation with the department of infectious diseases. After 4 weeks of treatment, the patient was discharged, since he did not present with clinical symptoms of infection and other symptoms had improved. On the 6-month follow-up MRI, the previously observed abscess was in good condition without recurrence, and there were no additional signs of symptom deterioration in the shoulder (Fig. 4A and 4B).

\section{Discussion And Conclusions}

Subscapular abscess is a rare surgical diagnosis, where a collection of pus forms between the subscapularis muscle and the chest wall. It has been described in only a few case reports. Predisposing conditions for this type of abscess include an immunocompromised state, recent infection, diabetes, endstage renal disease, IV drug use, and trauma to the shoulder area causing hematoma formation [1-4]. In a subscapular abscess, $S$. aureus is most commonly identified. The organism was $S$. aureus in four cases [1-4], methicillin-resistant $S$. aureus in three cases [5-7], Panton-Valentine leucocidin positive $S$. aureus in one case [8], H. influenza in one case [9], and no organism was grown in the last case [11].

To the best of our knowledge, this is the first report of a subscapular abscess caused by $E$. coli, which is a common gram-negative bacterium and the most common cause of bloodstream infection. However, the 
epidemiology has not been well defined in the non-selected population. A cohort study showed that infants and elderly were at highest risk for E. coli infection [12]. Hematogenous transmissions of $E$. coli usually occur as a complication of focal infections of the urinary or gastrointestinal tracts, but occasionally also can occur as IV drug use [13].

Our patient did not have any known immunocompromising condition, diabetes, or renal disease. However, he had been treated with IV antibiotics for six weeks for recent pneumonia in the upper left lobe of the lung. The cultures from the abscess fluid as well as blood cultures obtained the time of admission revealed extended-spectrum beta-lactamases (-) E. coli. However, urinalysis and urine culture results were negative. We believe that the patient might have been vulnerable to transient bacteremia, allowed translocation of E.coli from the IV injection route, ultimately resulting in an E.coli subscapular abscess.

Meanwhile, manual therapy and exercise, usually delivered together as components of a physical therapy intervention, are commonly used interventions for frozen shoulder. It includes any clinician-applied movement of the joints and other structures, for example, mobilization or manipulation. Mobilization is employed to reduce pain by stimulating peripheral mechanoreceptors and inhibiting nociceptors, and to increase joint mobility by enhancing exchange between synovial fluid and cartilage matrix [14, 15]. Manual therapy and stretching exercises have been demonstrated to reduce pain and improve function in a frozen shoulder [10]. Düzgün et al.[16] reported that manual therapy may be safely applied in frozen shoulder. He also suggested that manual therapy has a positive effect on pain, range of motion, muscular strength and level of the functional activity on frozen shoulders following intervention by physiotherapists. However, manual or massage therapy can cause complications, such as muscle bleeding, muscle rupture, or myositis ossificans $[17,18]$. Sasanuma et al.[19] reported that MRI in patients with severe frozen shoulder after manipulation showed capsule tears, labrum tears, and bone bruises in the humeral head.

In present case, the patient complained of experiencing severe pain with popping sound during a manual therapy of the left shoulder for frozen shoulder. MRI was observed with abscess in subscapularis. The minor trauma caused by manual therapy in severe frozen shoulder may have led to a small hematoma in the subscapular space that subsequently was seeded by extended-spectrum beta-lactamases (-) E. coli.

In summary, hematogenous transmissions of E. coli may occur as a complication of long-term intravenous drug use. Surgeons and physiotherapists should pay attention to the subscapular abscess as a rare complication of manual therapy in severe frozen shoulder.

\section{Abbreviations}

IV: Intravenous; S. aureus: Staphylococcus aureus, H. influenza: Haemophilus Influenzae; E. coli: Escherichia coli; MRI: Magnetic resonance imaging;

\section{Declarations}




\section{- Ethics approval and consent to participate}

Not applicable

\section{- Consent for publication}

Written informed consent was obtained from the patients for publication of their clinical datails and clinical images.

\section{- Availability of data and materials}

This is a case report of a single patient; in order to protect privacy and respect confidentiality, no part of the raw data has been made available in any public repository. The original operation reports, intraoperative photographs, imaging studies and outpatient clinic records were retained among the medical records of our institution per the normal procedure. All data concerning the case are presented in the manuscript.

\section{- Competing interests}

The authors declare that they have no competing interests.

\section{- Funding}

None.

\section{- Authors' contributions}

SIW designed this study. DHH participated in data collection, analysis and interpretation of data. $\mathrm{DHH}$ and SIW were the main contributors in writing this manuscript. All authors read and approved the final manuscript.

\section{- Acknowledgements}

None.

\section{References}

1. Handorf CR. Fatal subscapular staphylococcal abscess. South Med J. 1983;76(2):271. 
2. Nowinski RJ, Duchene C. Spontaneous septic subscapular abscess. A case report. J Bone Joint Surg Am. 2004;86(6):1302-4.

3. Saxena P, Konstantinov IE, Zelei D, Newman MA. Spontaneous subscapular abscess: a rare surgical condition. Heart Lung Circ. 2008;17(6):517-8.

4. Mourkus $H$, Vadivelu R, Phillips J. Literature review and a case report of spontaneous subscapular abscess in a child. Eur J Orthop Surg Traumatol. 2018;28(6):1235-40.

5. Giugale JM, Bosch PP, Grudziak JS. Subscapular Abscess in a Nine-Year-Old Female Patient: A Case Report. JBJS Case Connect. 2015;5(1):e13.

6. Koratala A, Alquadan KF, Chornyy V, Qadri I, Ejaz AA. Subscapular abscess associated with buttonhole cannulation technique of arteriovenous fistula for hemodialysis access. J Vasc Access. 2017;18(2):e18-9.

7. Christman-Skieller C, Mclntyre LK, Plevin R, Friedrich JB, Smith DG. A Posterolateral Approach to the Scapula for Evacuation of a Subscapular Abscess: A Case Report. JBJS Case Connect. 2017;7(3):e57.

8. Patel K, Spowart E, Sochorova D, Diego N, Mamarelis G, Sohail MZ. Subscapular Abscess Caused by Panton-Valentine Leukocidin-Positive Staphylococcus aureus: An Atypical Presentation. Case Rep Orthop. 2018;2018:8256428.

9. San Joaquin VH, Kimball JB. Subscapular abscess due to Haemophilus influenzae type B. Pediatrics. 1980;65(2):331-2.

10. Dueñas L, Balasch-Bernat M, Aguilar-Rodríguez M, Struyf F, Meeus M, Lluch E. A Manual Therapy and Home Stretching Program in Patients With Primary Frozen Shoulder Contracture Syndrome: A Case Series. J Orthop Sports Phys Ther. 2019;49(3):192-201.

11. Babayiğit A, Makay B, Demircioğlu F, Cakmakçi H, Unsal E. Subscapular abscess after blunt trauma. Pediatr Emerg Care. 2009 Jun;25(6):399-400.

12. Laupland KB, Gregson DB, Church DL, Ross T, Pitout JD. Incidence, risk factors and outcomes of Escherichia coli bloodstream infections in a large Canadian region. Clin Microbiol Infect. 2008;14(11):1041-7.

13. Chang CJ, Chang WN, Huang LT, Huang SC, Chang YC, Hung PL, Lu CH, Chang CS, Cheng BC, Lee PY, Wang KW, Chang HW. Bacterial meningitis in infants: the epidemiology, clinical features, and prognostic factors. Brain Dev. 2004 Apr;26(3):168-75.

14. Frank C, Akeson WH, Woo SL, Amiel D, Couts RD. Physiology and therapeutic value of passive joint motion. Clinical Orthopaedics and Related Research 1984;185:113-25.

15. Vermeulen HM, Obermann WR, Burger BJ, Kok GJ, Rozing PM, Ende $\mathrm{CH}$. End-range mobilization techniques in adhesive capsulitis of the shoulder joint: a multiple-subject case report. Physical Therapy 2000;80:1204-13.

16. Düzgün I, Baltaci G, Atay OA . Manual therapy is an effective treatment for frozen shoulder in diabetics: an observational study. Eklem Hastalik Cerrahisi. 2012;23(2):94-9. 
17. Tzaveas A, Anastasopoulos N, Paraskevas G, Natsis K. A Rare Case of Quadratus Femoris Muscle Rupture After Yoga Exercises. Clin J Sport Med. 2016;26(5):e105-7.

18. Wei J, Jia Y, Liang B. Myositis ossificans of the serratus anterior as a rare complication of massage: a case report. J Med Case Rep. 2015 Jun 16;9:143.

19. Sasanuma $H$, Sugimoto $H$, Kanaya $Y$, et al. Magnetic resonance imaging and short-term clinical results of severe frozen shoulder treated with manipulation under ultrasound-guided cervical nerve root block. J Shoulder Elb Surg. 2016;25(1):e13-20.

\section{Figures}

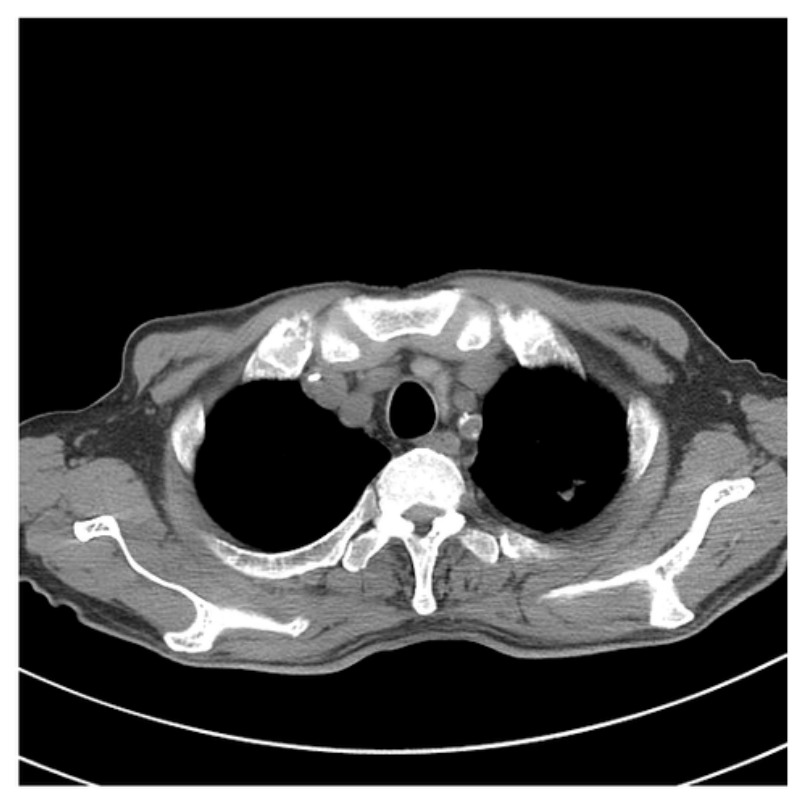

a

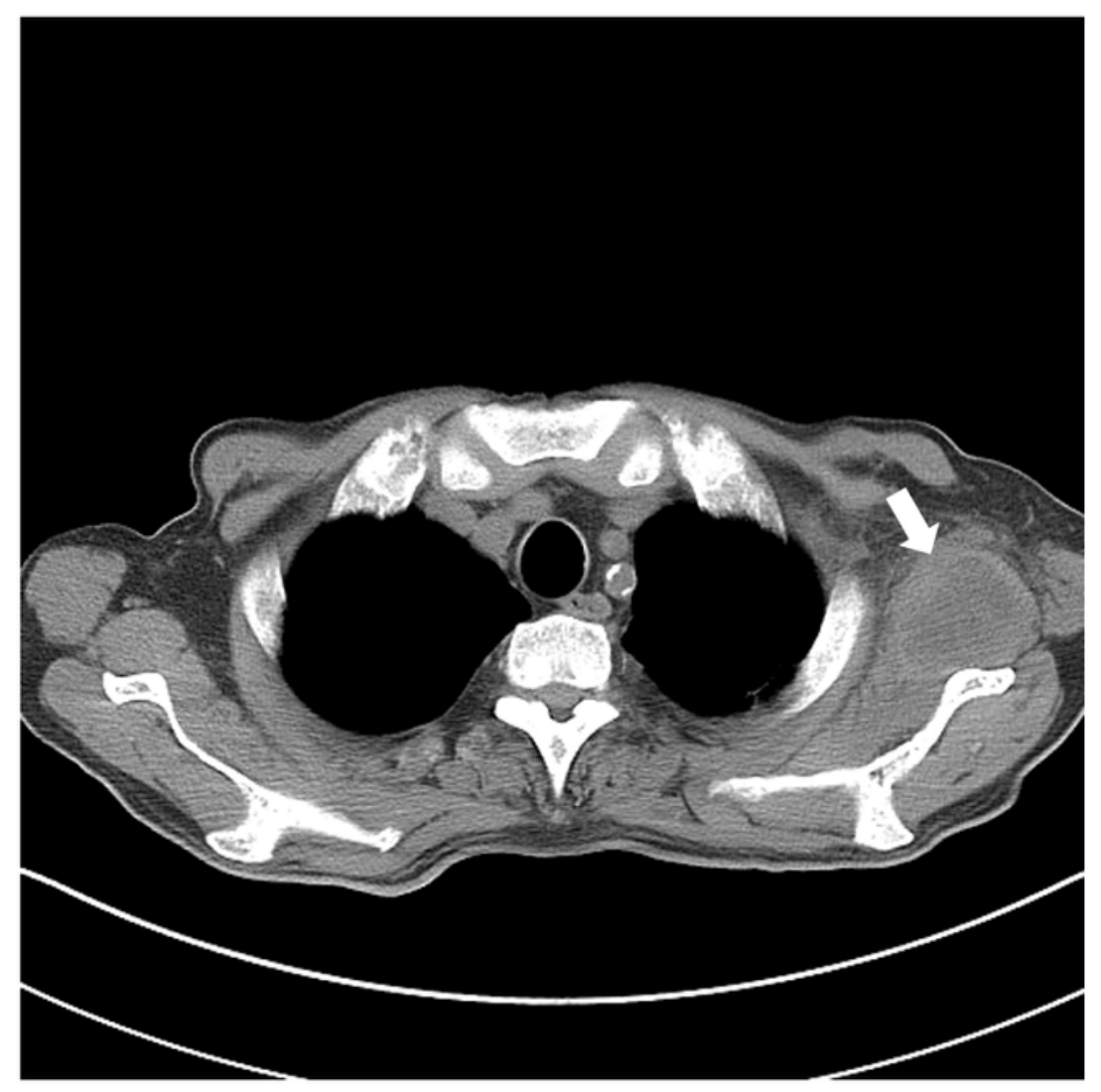

b

\section{Figure 1}

Chest computed tomography (CT) scan of a 72-year-old male with left shoulder pain. A: CT scan taken at internal medicine before referral showing improvement of previous pneumonia and no lesions around the shoulder B: CT scan obtained after 4 weeks showing newly observed lesion with thickened walls in the left subscapularis muscle, including fluid in an area of $10 \times 5$ (white arrow). 

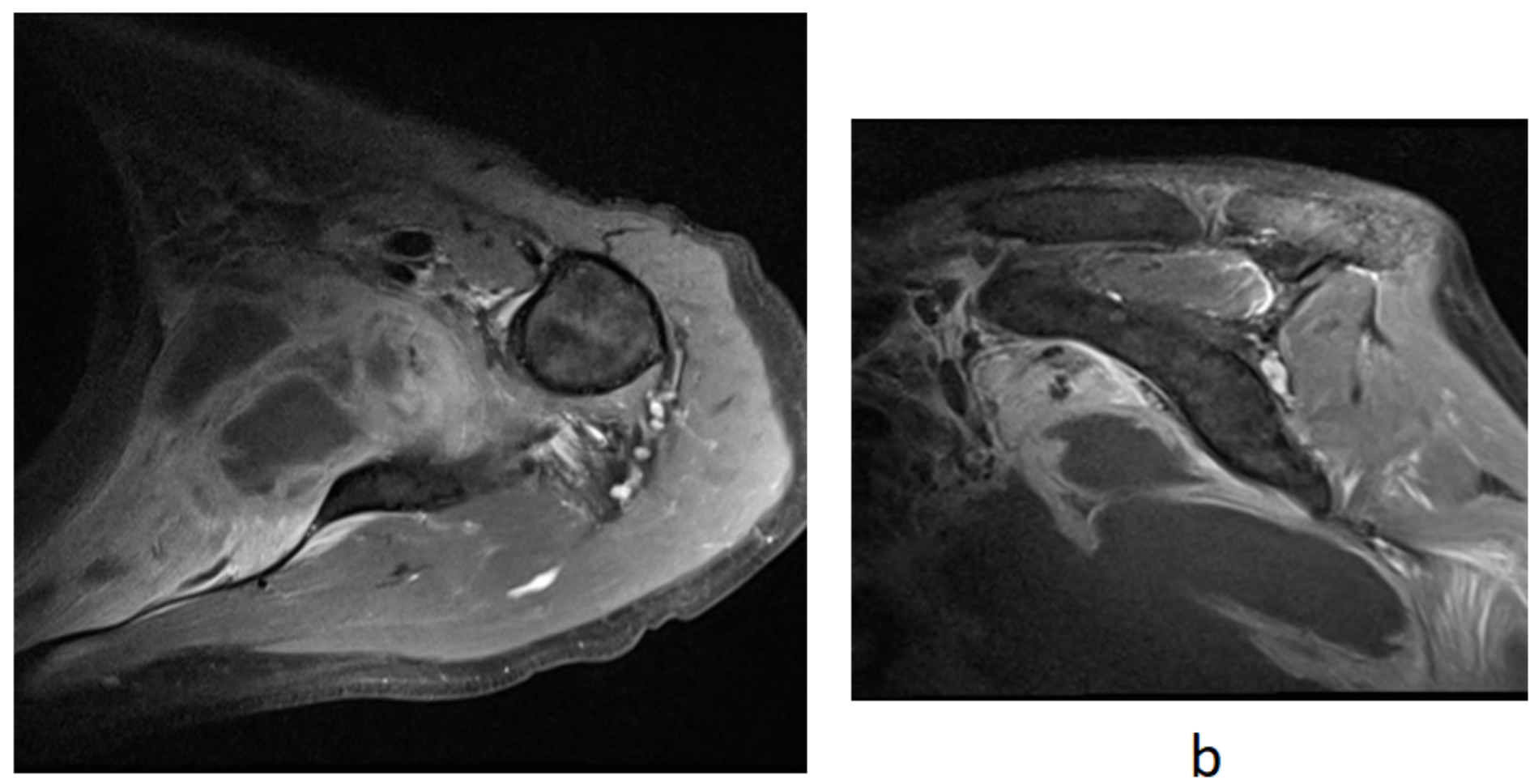

a

b

\section{Figure 2}

2A and 2B: Contrast-enhanced magnetic resonance (MR) imaging of left shoulder Axial and sagittal fatsuppressed, T2-weighted images showing large intramuscular complicated fluid collection at the level of the subscapularis muscle $10.0 \times 5.2 \times 11 \mathrm{~cm}$ in size 


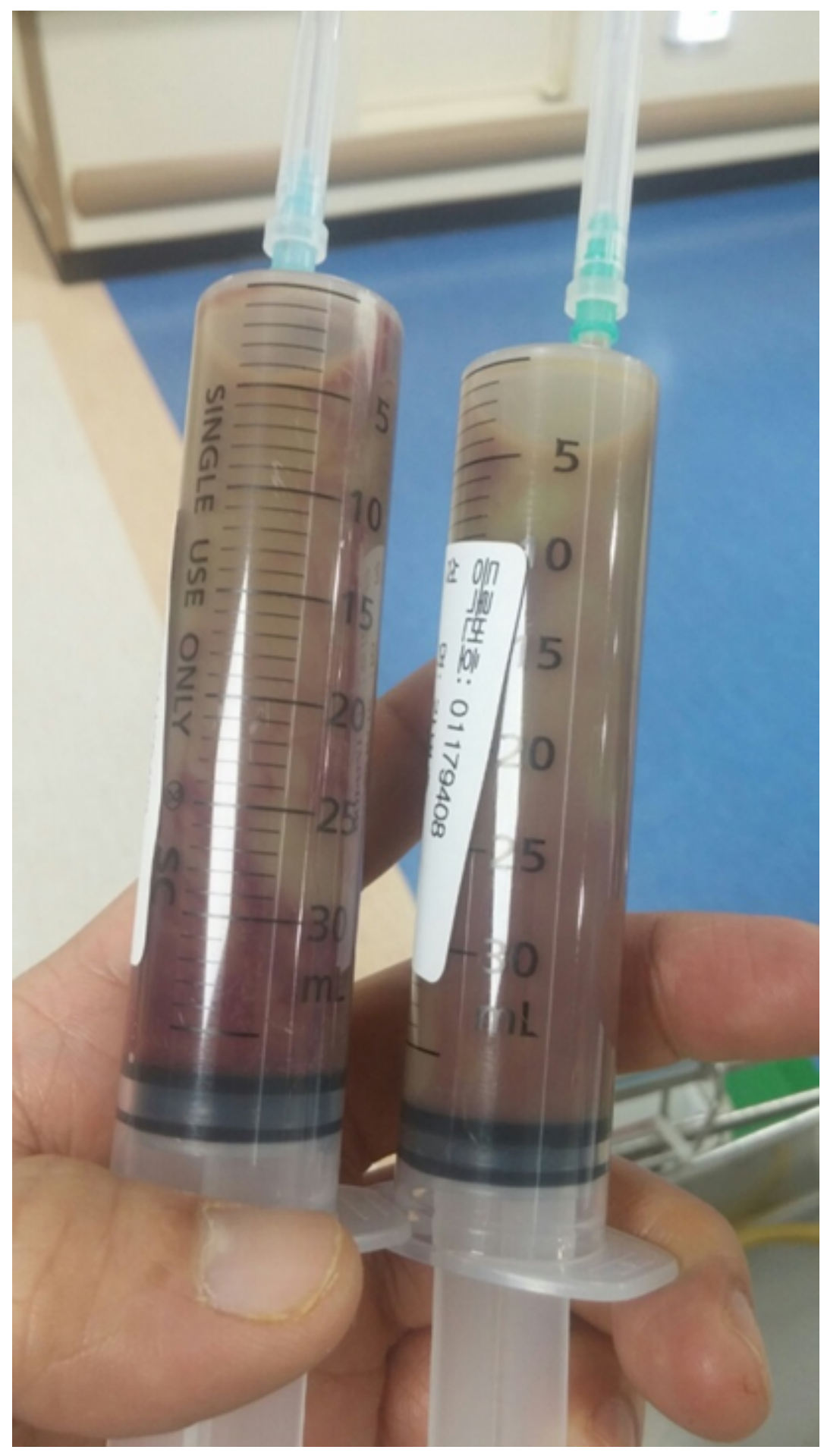

\section{Figure 3}

Aspiration showing approximately $70 \mathrm{ml}$ of bloody hematoma and turbid pus-like discharge. 

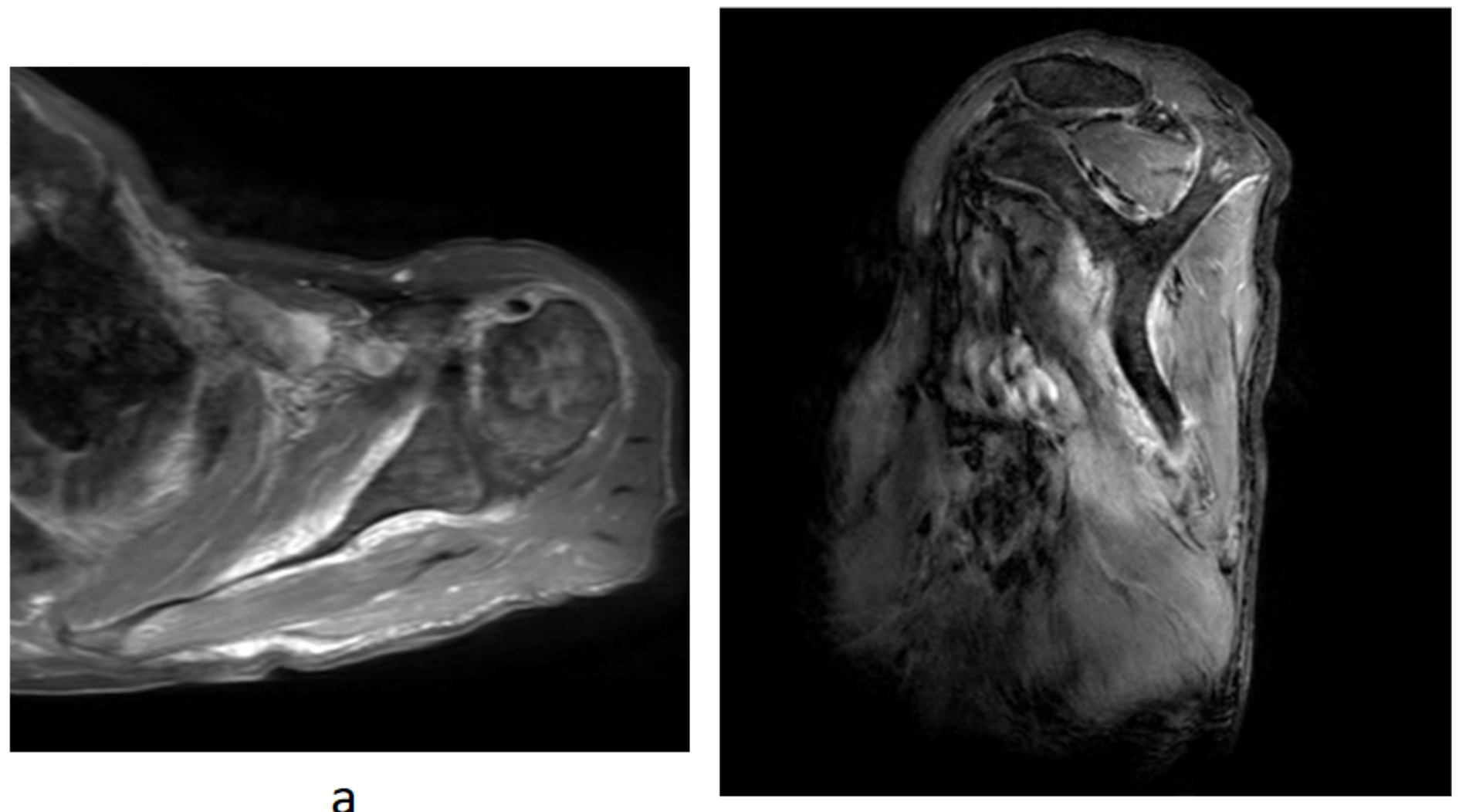

a

b

\section{Figure 4}

4A and 4B: Contrast-enhanced MR imaging obtained at 6 months after surgical drainage; previously observed abscess is in good condition without recurrence. 